\title{
Pragas quarentenárias florestais: riscos e prevenção
}

\author{
Edson TAdeu IEDE \\ Susete do Rocio Chiarello Penteado \\ WILSON REIS FILHO
}

\section{INTRODUÇÃO}

A economia brasileira até o início da década de 90, apresentava-se praticamente fechada ao comércio internacional. Com a abertura econômica e a globalização, houve um aumento substancial na movimentação de mercadorias, inclusive de produtos de origem vegetal. Incrementou-se também, o turismo internacional e esta associação de fatores, aliada à falta de estrutura da defesa fitossanitária, propiciaram um aumento do risco de introdução de pragas exóticas. Estes riscos são potencializados quando se referem às pragas florestais. Estas, além de serem veiculadas em materiais de propagação (sementes, mudas, estacas, etc.), em madeiras em toras e serradas, são transportadas, principalmente, em madeiras de embalagem e suporte de mercadorias, usadas na acomodação de cargas em diferentes meios de transporte. Estas madeiras, normalmente são de baixa qualidade, e devido ao grande volume, são difíceis de serem inspecionadas pelos serviços quarentenários. (IEDE \& PENTEADO, 2000).

A existência de extensas áreas contínuas de reflorestamentos no Brasil, principalmente com espécies de Pinus (2,0 milhões de ha) e de Eucalyptus (3,2 milhões de ha), normalmente com uma base restrita de espécies e procedências, oferecem condições propícias para o estabelecimento e dispersão de pragas exóticas.

A baixa resistência ambiental devido aos monocultivos, que não oferecem condições de abrigo e/ou alimentos durante as fases de desenvolvimento de inimigos naturais, assim como, a própria ausência desses em ambientes onde a praga exótica foi introduzida, propiciam condições para uma rápida explosão populacional e dispersão do organismo invasor.

Face aos riscos citados, foram listadas, através de diferentes métodos de análise de riscos de pragas, várias espécies que são potencialmente quarentenárias para o país, assim como, materiais de risco de introdução destas espécies. 


\section{PLANTIOS FLORESTAIS E OS INDICADORES DE RISCO}

Com a destruição das florestas nativas e devido às dificuldades para recompor estes ecossistemas originais, além dos problemas de pragas e doenças que inviabilizam técnica e economicamente os reflorestamentos homogêneos com as espécie nativas, optou-se pela introdução de espécies florestais exóticas. Para atender à demanda de madeira para diversos fins, iniciaram-se há mais de 40 anos, no Brasil, projetos de reflorestamento com a introdução de espécies, principalmente, dos gêneros Pinus e Eucalyptus.

Mais recentemente foram introduzidas espécies da família Salicaceae. Os plantios comerciais de Populus spp. foram estabelecidos visando o fornecimento de matéria prima alternativa para a fabricação de fósforos. Por outro lado, existe a espécie nativa, Salix humboldtiana, que está presente em quase todo o país, abrangendo as mais diversas regiões edafoclimáticas.

Diversas espécies do gênero Populus estão amplamente distribuídas, em forma de árvores ornamentais e cortinas quebra-ventos. Além disso, há uma busca constante por espécies exóticas alternativas que apresentem uma capacidade de adaptação às diferentes regiões bioclimáticas.

Em função da crescente demanda interna de madeira, assim como, para atender as metas de exportação destes produtos, fez-se necessário aumentar a área reflorestada, utilizar técnicas para atingir altas produtividades e estabelecer uma melhoria no padrão genético destas espécies florestais.

A introdução, estabelecimento e dispersão de pragas exóticas estão associados à presença de alguns indicadores de risco como, plantações monoespecíficas, com alta densidade de plantas, plantios mal manejados ou localizados em sítios ruins, utilização de técnicas para atingir alta produtividade (plantios clonais), entre outros.

Sem considerar-se os aspectos dos riscos propiciados pelo aumento substancial do comércio internacional nos últimos anos, estes cenários demonstram que, gradativamente, vêm aumentando o risco de introdução, estabelecimento e dispersão de pragas exóticas no país.

\section{PRAGAS EXÓTICAS - FORMAS DE INTRODUÇÃO E IMPACTOS}

Pragas e doenças podem ser introduzidas em novos ambientes por várias maneiras. CIESLA (1993) afirma que o homem é um fator importante, tanto como vetor direto, como no comércio internacional ou na movimentação de materiais vegetais. A introdução de plantas exóticas em novos habitats, especialmente na forma de monocultivos extensivos, propicia condições ideais para a colonização e o estabelecimento de uma nova praga introduzida, assim como, facilitam a sua dispersão. 
Dentre as mercadorias de origem florestal, a madeira não processada, especialmente em toras, são materiais apropriados para a introdução de muitas espécies de besouros de casca e brocas da madeira. Madeiras utilizadas para acomodação de cargas, nos diferentes meios de transporte, assim como, a madeira de paletes e de embalagens (caixas, caixões, carretéis, etc.), são materiais de alto risco. Normalmente estes materiais são fabricados com madeira de baixa qualidade, ou de sobras de madeira, que muitas vezes contêm restos de casca (Iede \& Penteado, 2000).

WALLNER (1996) considera que pragas invasoras ou "poluentes biológicos", são muito perigosos para a diversidade biológica em um ecossistema florestal. Eles podem causar alterações nas práticas de manejo florestal, perdas econômicas e impactos ambientais indesejáveis. As pragas invasoras normalmente colocam em risco a estabilidade e produtividade dos ecossistemas. $\mathrm{Na}$ ausência de inimigos naturais, que regulam estas pragas no local de origem, eles aumentam sua população rapidamente. Mesmo insetos que na origem, não têm importância econômica, ou são considerados secundários, quando introduzidos em novas áreas, provocam perdas econômicas significativas devido à ausência de mecanismos biológicos que regulem a população.

Os danos provocados por estes insetos introduzidos vão desde perdas econômicas até consequiências ambientais desastrosas como: desflorestamento, simplificação do ecossistema, destruição da vida selvagem e de habitat ripários e aumento dos riscos de incêndio (WALLNER, 1996). Além disso, pode causar mudanças na composição da flora, perturbar as atividades recreacionais, depreciar o valor de imóveis rurais e/ou residenciais e afetar a saúde humana.

As perdas provocadas pelo ataque de pragas às árvores dependem da espécie da praga, do tipo de dano que causa, do valor comercial da madeira. Deve-se estimar também os custos de remoção das plantas mortas, custos de controle e custos de replantio. Além disso, as pragas exóticas, muitas vezes são quarentenárias para outros países que mantém relações comerciais com o país em que ela foi introduzida. Em função disso, o país importador pode impor barreiras fitossanitárias, causando entraves no comércio que vão desde o aumento do preço da mercadoria, até a impossibilidade de sua comercialização, devido a tratamentos fitossanitários. Não havendo comércio da madeira e de seus derivados, esta não é explorada no campo, acarretando problemas sociais como o desemprego e suas implicações, além de aumentar o risco de ataque de outras pragas ou o incremento da população da praga introduzida, face ao estresse propiciado pela super estocagem da madeira no campo (IEDE et al. 2000). Normalmente estes prejuízos paralelos às perdas no campo não são considerados pelos produtores e economistas.

De acordo com PIMENTEL (1986), das 70 principais pragas florestais nos Estados Unidos, 19 haviam sido introduzidas, ou seja, 27\%. Em termos 
econômicos, as perdas provocadas por insetos introduzidas nos Estados Unidos, no período de 1906/1991, foram estimadas em 92 bilhões de dólares (Office of Technology Assesment, 1993 citado por WINDLE, 1997).

\section{ESPÉCIES FLORESTAIS E PRAGAS EXÓTICAS INTRODUZIDAS}

\section{Eucalyptus spp.}

As áreas reflorestadas com o gênero Eucalyptus estão entre as mais importantes no Brasil, com cerca de 3,2 milhões de ha, plantados com diferentes espécies, adaptadas as mais variadas condições edafoclimáticas, de Norte à Sul do país. Destacam-se como as mais plantadas, entre as 92 espécies introduzidas no Brasil: E. alba, E. camaldulensis, E. cloeziana, E. deanei, E. dunnii, E. grandis, E. maculata, E. microcorys, E. pellita, E. pilularis, E. saligna, E. tereticornis, E. urophylla e E. viminalis. E. sideroxilon e E. viminalis.

Grande parte dos reflorestamentos com Eucalyptus expandiu-se por regiões com limitações de solo, déficit hídrico e ocorrência de geadas, através da utilização de espécies e procedências mais adequadas para situações ambientais específicas.

As condições edafoclimáticas existentes no país são bastante favoráveis a dispersão das pragas originárias, principalmente da Australásia, havendo inclusive condições de grandes áreas contínuas de plantios de Eucalyptus que favorecem a rápida dispersão.

No Brasil, as pragas florestais introduzidas de maior importância, foram primeiramente aquelas de Eucalyptus spp., como os gorgulhos Gonipterus gibberus e G. scutellatus (Coleptera: Curculionidae), a broca Phoracantha semipunctata (Coleoptera: Cerambycidae) e mais recentemente, na década de 1990, Ctenarytaina eucaliptii, C. spatulata e Blastopsylla occidentalis (Homoptera: Psyllidae) (IEDE et al. 1997; BURCKHARDT et al. 1999).

\section{Pinus spp.}

Os plantios de Pinus spp estão concentrados em uma área de dois milhões de hectares, com a finalidade de fornecer madeira para a produção de papel e celulose, de madeira serrada e de maneira para laminação.

A ampla variação na adaptabilidade e crescimento das espécies do gênero Pinus permite ganhos elevados em produtividade, e aquelas originárias do sul dos Estados Unidos ( $P$. taeda e $P$. elliottii) estão sendo muito utilizadas na região Sul do Brasil. Na região tropical do Brasil, espécies como P. oocarpa, $P$. caribaea caribaea, $P$. caribaea hondurensis, $P$. caribaea bahamensis, têm sido plantadas com a finalidade de produzir madeira para serraria e laminação. 
Os plantios de Pinus spp., no Brasil, após um período bastante longo, livre de pragas, com a introdução da vespa-da-madeira, Sirex noctilio em 1988, passou a ter sua produtividade ameaçada pelos ataques da praga, inclusive colocando em risco este extenso patrimônio florestal brasileiro. Entretanto, com as medidas adotadas pelo Programa Nacional de Controle à Vespa-da-Madeira (PNCVM), com o apoio do Fundo Nacional de Controle à Vespa-da-Madeira (FUNCEMA), está sendo possível conviver com a praga de modo que esta não venha a comprometer os plantios de Pinus spp. do país. Na década de 90, foi registradas a presença de espécies exóticas de pulgões do gênero Cinara (C. maritima, C. atlantica, C. pinivora), Eulachnus rileyi e Essigella californica atacando plantios de Pinus spp.(LAZZARI \& ZONTA-DE-CARVALHO 2000).

A introdução destas espécies causou perdas econômicas significativas aos reflorestadores, exigindo-se a elaboração de programas de controle destas pragas, aumentando-se substancialmente os custos de produção.

A fim de estabelecer uma melhoria no padrão genético das espécies de Pinus presentes na região, assim como, para definir espécies alternativas, que apresentem uma capacidade de adaptação às diferentes condições ecológicas, são mantidos programas constantes de introdução e reintrodução de material genético. Esta situação pode levar ao risco de introdução de pragas exóticas nas regiões de cultivo destas espécies.

\section{Salicaceae}

No Brasil os plantios comerciais de Populus spp. são mais recentes, a fim de servir como matéria prima alternativa para a fabricação de fósforos. As maiores áreas reflorestadas estão localizadas no sul do Paraná. Por outro lado, há uma espécie nativa, Salix humboldtiana, que está presente em grande parte do Brasil, abrangendo as mais diversas regiões bioclimáticas e edáficas, distribuindo-se desde o clima tropical úmido da Amazônia até as regiões de clima subtropical e temperado do Sul. Além disso, diversas espécies do gênero Populus estão amplamente distribuídas na Região, em forma de árvores ornamentais, cortinas quebra-ventos e como citado anteriormente, em plantios comerciais.

Devido à plasticidade tanto da espécie nativa como das espécies introduzidas (Salix spp. e Populus spp.) em parte significativa da região, desde que introduzida alguma praga, esta terá as mais variadas alternativas para adequar-se ao ambiente, da região.V. ANÁLISE DE RISCO DE PRAGAS (ARP)

Para que se conheça sobre o risco de introdução de uma certa mercadoria, ou sobre o estatus quarentenário de uma praga é necessário que se faça uma análise de risco do material ou da praga. Apoiado por uma base de dados, constituída por registros de interceptações de pragas exóticas, pelos 
Serviços de Defesa Fitossanitária de diferentes países e por dados técnicos científicos, deve-se avaliar o risco de introdução de pragas, para a área em perigo.

Com auxilio de critérios geográficos e regulamentareis, avalia-se o potencial de adaptação da praga segundo suas exigências climáticas e geográficas. Com base nos danos causados em outros países, onde a praga está presente, deve-se avaliar a sua importância econômica. Através de dados a respeito da presença de hospedeiros na região em perigo, da abundância e continuidade destes plantios, avalia-se o potencial de estabelecimento, o potencial de dispersão após o estabelecimento da praga e a importância econômica potencial, na região em perigo. Deve-se analisar, finalmente, o potencial de introdução da praga, através da análise das condições de transporte, do material que esta sendo introduzido, aliado ao comportamento da praga, entre outros. Em uma fase posterior deve-se estabelecer o Manejo de Risco, a fim de estabelecer requisitos fitossanitários, para mitigar o risco de introdução da praga, na área em perigo.

O Grupo de Trabalho Permanente em Sanidade Silviagrícola (GTPSSA), do Comitê de Sanidade Vegetal do Cone Sul (COSAVE) realizou uma ARP expedita, selecionando pragas potencialmente quarentenárias dos gêneros Pinus, Eucalyptus e da família Salicaceae, em função da importância econômica destas espécies florestais, na região dos países do COSAVE. O GTPSSA considerou que os materiais de embalagem e suporte de mercadorias fabricados com madeira, utilizados no transporte internacional, como caixas, caixões, carretéis, paletes, madeira de estiva, etc. são de alto risco, por constituírem-se em um meio eficaz de disseminação de pragas a grandes distâncias, particularmente aquelas que atacam espécies florestais. Isto, em função da baixa qualidade da madeira utilizada para estes fins, e a dificuldade de se fazer inspeções quarentenárias, tanto pelo volume de material, como pela falta de estrutura de fiscalização dos serviços de defesa fitossanitária da maioria dos países. Além disso, havia registro de várias interceptações de pragas de importância quarentenária, realizadas no Chile, em embalagens de madeira, assim como estas são reconhecidas como materiais de alto risco por outras organizações como o USDA-APHIS, o NASS da Nova Zelândia e o AQUIS da Austrália,

Por estes aspectos, o grupo sugeriu que se implementasse, no menor tempo possível, o marco legal que permitisse aos Serviços Nacionais de Sanidade Vegetal, elaborar as estratégias mais adequadas para a fiscalização destes materiais. Este marco legal deveria contemplar os seguintes aspectos:

1) que as madeiras de embalagem e suporte ingressem livres de casca, de pragas e sem danos aparentes causadas por pragas; ou então que a madeira tenha sido seca em estufa (kiln dry), ou tratada com preservativos, ou fumigantes devidamente autorizados. 
2) que se estabeleça de forma clara e precisa, a faculdade do Serviço Nacional de Defesa Fitossanitária para inspecionar qualquer tipo de embalagem e suporte de madeira, utilizados no transporte e comércio internacional de mercadorias, assim como para ordenar tratamentos quarentenários, ante somente a presença de danos e aplicar outras disposições que sejam pertinentes.

Baseados ainda na análise expedita, iniciou-se a elaboração de regulações quarentenárias para a importação de sementes, plantas e partes de plantas, madeira não processada e para materiais de embalagem e suporte de madeira. Além disso, baseados num standart regionais foram e estão sendo realizadas análises de risco para diferentes pragas e doenças exóticas que atacam as espécies florestais selecionadas na primeira fase.

\section{PRAGAS QUARENTENÁRIAS FLORESTAIS}

Como resultado das análises de risco de pragas, foram elaborados os listados de pragas quarentenárias florestais A1 e A2 para a região do COSAVE.

Considerando-se as definições de pragas A1 e A2 da Food Agricultural Organization (FAO) sendo as pragas A1, aquelas ausentes da região e as pragas $\mathrm{A} 2$, aquelas presentes na região, porém com distribuição restrita e sob controle oficial.

Para as espécies de Pinus, são considerados de importância quarentenária os Scolytidae dos gêneros Dendroctonus spp. e Ips spp.; o gorgulho da família Curculionidae, Hylobius abieties; os Cerambycidae Anoplophora spp, e Monochamus spp., sendo, este último, vetor do nematóide Bursaphelenchus xylophilus. Com relação aos lepidópteros desfolhadores, apresentam-se como quarentenárias para o Brasil, as mariposas Lymanthria dispar e L. monacha, a processionária do pinus Thaumetopoea pityocampa e os Diprionidae, Diprion spp. e Neodiprion sertifer. Com relação à semente, podese considerar de risco Megastigmus spp. (Hymenoptera). Os patógenos considerados de importância quarentenária são: Scirrhia acícula, Peridermium spp., Cercoseptoria pini-densiflorae e Cronartium spp.

Até o momento, das pragas analisadas, cujos hospedeiros são Eucalyptus spp., nenhuma foi considerada de importância quarentenária.

Através de processos de análise de risco de pragas de Salicaceae foram identificadas como quarentenárias: Saperda carcharias e $S$. populnea (Cerambycidae); Cryptorrynchus lapathi (Curculionidae); Helicomya saliciperda (Cecidomiydae); Paranthrene tabaniformis (Lepidoptera) e as bactérias Aplanobacter populi e Erwinia salicis. 
A introdução dessas pragas no país não representam apenas os danos diretos causados por elas nas plantas florestais, mas sim nos seus efeitos cumulativos, como perda de clientes ou mesmo fechamento do comércio internacional do produto, custos para desenvolvimento de pesquisas, implantação e execução de medidas quarentenárias (barreiras, vigilância) e controle.

\section{BIBLIOGRAFIA}

BURCKHARDT, D.; SANTANA, D. L. Q.; TIERRA, A. L.; ANDRADE, F.M.; PENTEADO, S.R.C., IEDE, E.T.; MOREY, C. Psyllid pests (Hemiptera: Psyllidae) in South America eucalypt plantations. (in press).

CIESLA, W.M. (1993) Recent Introductions of Forest Insects and their Effects: A Worldwide Overview. In: Conferência Regional da Vespa-da-madeira, Sirex noctilio, na América do Sul.(1992: Florianópolis). Anais. ColomboEMBRAPA/FAO/FUNCEMA..p. 09-21.

IEDE, E. T.; LEITE, M.S.P.; PENTEADO, S.R.C.; MAIA, F. (1997). Ctenarytaina sp. (Homoptera: Psyllidae) associada a plantios de Eucalyptus sp. em Arapoti, PR. In: Congresso Brasileiro de Entomologia. (16.: Salvador) Resumos do Congresso Brasileiro de Entomologia/-Salvador: Sociedade Entomológica do Brasil, 1998. p. 253.

IEDE, E. T.; LAZZARI, S. M.; PENTEADO, S.R.C.; ZONTA-deCARVALHO, R.C.; TRENTINI, R.F.R. (1998). Ocorrência de Cinara pinivora (Homoptera: Aphididae) em Pinus elliottii e P. taeda em Cambará do Sul (RS) e Lages (SC). In: Congresso Brasileiro de Zoologia, (12.: Recife). Resumos do Congresso Brasileiro de Zoologia/-- Recife: Sociedade Brasileira de Zoologia. 1998. p. 141.

IEDE, E. T. \& PENTEADO, S.R.C. (2000) Detecção e controle de pragas quarentenárias - Sirex noctilio no Brasil. In: Silvotecna (9.: Concepción). Proceedings. da Silvotecna. Concepción: Corporación Chilena de la Madera, 2000. 1 CD- ROM.

IEDE, E. T.; REIS FILHO, W. \& PENTEADO, S.R.C. Pragas quarentenárias florestais. In: Encontro de Fitossanitaristas, (.:Foz do Iguaçu, 2000), Anais do Encontro Nacional de Fitossanitaristas/-- Brasília: Ministério da Agricultura e do Abastecimento, 2000. 1 CD-ROM.

PIMENTEL, D. (1986) Biological invasions of plants and animals in agriculture and forestry. In: Ecology of Biological Invasions of North America and Hawaii (eds Mooney, H.A. \& Drake, J.A.), pp. 149162.Springer-Velag, New York (US). 
WALLNER, W.E. (1996) Invasive pests ('biological pollutants') and US forests: whose problem, who pays? Bulletin OEPP/EPPO, Bulletin 26, 167-180.

WINDLE, P.N., (1997) The Ota Report on Harmful Nonindigenous Species. In: Conference of Exotic Pests of Eastern Forests. (Nashville). Proceedings of Conference of Exotic Pests of Eastern Forests/-- Nashville: USDA FOREST SERVICE/TN EXOTIC PEST PLANT COUNCIL, 1997. p. 7176. 